\title{
La inteligencia emocional: un camino para la resolución de conflictos en Instituciones Educativas Rurales, Rionegro, Colombia
}

Emotional intelligence: A path for conflict resolution inRural Educational

Institutions, Rionegro, Colombia

Inteligência emocional: Um caminho para a resolução de conflitos em Instituições

Educacionais Rurais, Rionegro, Colômbia

\author{
NINY JOHANA SALAZAR GÓMEZ ${ }^{1}$ \\ DEIVIS ROBINSON MOSQUERA ALBORNOZ² \\ MARTHA CECILIA SÁNCHEZ SOLANO3
}

\begin{abstract}
RESUMEN
El objetivo del presente estudio se orienta a establecer la relación entre inteligencia emocional y los roles del maestro en la enseñanza y del estudiante en el aprendizaje para el manejo de situaciones de conflicto en los grados $6^{\circ}$ en Instituciones Educativas Rurales del municipio de Rionegro; este proyecto de investigación es de orden mixto, se fundamenta en el método inductivo, los métodos, representan un conjunto de procesos sistemáticos empíricos y críticos de investigación el cual articula los enfoques cualitativo y cuantitativo para desarrollar el estudio de los significados de las acciones humanas y de la vida social, esto se revela a través del contacto con los docentes mediante una entrevista y la interacción directa de los estudiantes en un taller investigativo. Se pretende comprender que cuando se fortalece la inteligencia emocional en la escuela mejoran los procesos de aprendizaje emocionales sociales y se adquieren habilidades para la vida.
\end{abstract}

Palabras clave: Inteligencia; maestro; estudiante; proceso de aprendizaje; resolución de conflictos.

\footnotetext{
ABSTRACT

The objective of this study is aimed at establishing the relationship between emotional intelligence and the roles of the teacher in teaching and the student in learning, in order to manage conflict situations in sixth grades in Rural Educational Institutions in the municipality of Rionegro. This research project is of mixed order; it is based on the inductive method. The methods represent a set of systematic empirical and critical research processes, which articulates the qualitative and quantitative approaches to develop the study of the 
meanings of human actions and social life. This is revealed through contact with teachers through an interview and direct interaction of students in an investigative workshop. The aim is to understand that when emotional intelligence is strengthened at school, social emotional learning processes improve and life skills are acquired.

Keywords: Intelligence; teacher; student; learning process; conflict resolution.

\section{RESUMO}

O objetivo deste estudo é estabelecer a relação entre inteligência emocional e os papéis do professor no ensino e do aluno na aprendizagem de administrar situações de conflito nas sextas séries nas Instituições de Ensino Rural do município de Rionegro. Este projeto de pesquisa é de ordem mista, é baseado no método indutivo. Os métodos representam um conjunto de processos sistemáticos de pesquisa empírica e crítica, que articulam abordagens qualitativas e quantitativas para desenvolver o estudo dos significados das ações humanas e da vida social. Isso é revelado através do contato com os professores por meio de uma entrevista e interação direta dos alunos em uma oficina de investigação. O objetivo é entender que, quando a inteligência emocional é fortalecida na escola, os processos de aprendizado emocional social melhoram e as habilidades para a vida são adquiridas.

Palavras-chave: Inteligência; professor; aluna; processo de aprendizagem; resolução de conflitos.

\section{INTRODUCCIÓN}

Cantidad de investigaciones se han dedicado a la presente temática, especialmente en países como España y Estados Unidos, dejando resultados que aportan significativamente a cualquier trabajo que se desee emprender en cuanto a la inteligencia emocional, es por ello que en el marco de la definición que aporta (Goleman, 1999) y más adelante (Shapiro, 2001), se pretende establecer la relación entre inteligencia emocional y los roles del maestro en la enseñanza y del estudiante en el aprendizaje para el manejo de situaciones de conflicto en los grados $6^{\circ}$ en Instituciones Educativas Rurales del municipio de Rionegro.

En tanto la inteligencia emocional implica un estado de conciencia de las personas frente a las situaciones interactivas en su contexto, en los procesos intersubjetivos implica comprensión de dichas situaciones que interrelacionen razón y emoción, y posibilite desarrollar capacidad adaptativa para enfrentar los conflictos, para Correa (2018) estos "son una realidad inherente a la condición humana, se relacionan con hechos de nuestra vida y se producen en la interacción entre las personas, generando tensiones y estados emocionales que se reflejan en las actitudes"

Una de los temas que genera mayor preocupación en la escuela es el desarrollo intelectual de los estudiantes, el cual permea en un alto porcentaje el rendimiento académico. Con frecuencia, se implementan estrategias que llevan a los estudiantes a mejorar los niveles académicos que son tan evaluados por entidades externas a la escuela como el Ministerio de Educación Nacional. Sin embargo, con esta preocupación, se ha abandonado un tema de igual complejidad y trascendencia como lo es el desarrollo de la inteligencia emocional; 
definida por (Salovery, Brackett \& Mayer, 2004) como la habilidad de las personas para percibir (en uno mismo y en los demás) y expresar las emociones de forma apropiada, usando dicha información emocional para facilitar el pensamiento, para comprender y razonar sobre las emociones y regularlas en sí mismo e identificarlas en los demás; es decir, el uso adaptativo de las emociones de manera que el individuo pueda solucionar problemas y adaptarse de forma eficaz al medio que lo rodea. Este asunto se ha convertido en una llaga para la educación en la medida en que se cuenta en las aulas con estudiantes que no poseen habilidades necesarias para el manejo de las emociones. Evidencia de ello, las situaciones de conflicto que diariamente se presentan en los espacios de aprendizaje, donde el maestro quiere resolver la responsabilidad de orientar el conocimiento y los estudiantes interrumpen dicha actividad con agresiones físicas y verbales entre ellos e incluso con los mismos maestros.

Según (Goleman, 1999) las bases para el desarrollo de la inteligencia emocional se adquieren en la familia durante los primeros años de formación, pero la sociedad actual, muestra una transformación familiar que ha llevado a la des configuración de responsabilidades en la formación del individuo, a partir de los roles que se asumen en el trayecto de vida, asunto que se refleja todo el tiempo en la escuela, de ahí la preocupación por comprender ¿Cómo se asumen los roles en el desarrollo emocional, en situaciones de tolerancia y conflicto que se manifiestan en el aula, los maestros en la enseñanza y estudiantes en el aprendizaje? ¿Cuál es el papel de la escuela en el desarrollo de la inteligencia emocional en los escolares? ¿Cuál es la responsabilidad como agentes formadores en el afianzamiento de la inteligencia emocional? ¿Cómo se convierte el docente en un referente para el estudiante de acuerdo al manejo de sus emociones? Realmente son múltiples los cuestionamientos que surgen con el abordaje de tan relevante temática.

La vivencia en el contexto educativo de situaciones donde los estudiantes y hasta los mismos maestros se descompensan y toman reacciones no adecuadas en la solución de los conflictos cotidianos, deja entrever la necesidad de abordar el tema de la inteligencia emocional en la escuela como un problema que requiere atención ; diariamente se viven situaciones de conflicto y agresión entre estudiantes y en ocasiones con maestros, que deben ser resueltos por alguna de las vías que proporciona la legislación colombiana, teniendo muy presente que esas soluciones deben contribuir a la sana convivencia en las Instituciones. En la cotidianidad se viven momentos donde el estado emocional de los agentes que hacen parte del proceso educativo, se ve alterado revertiéndose esto, en acciones de agresión física, verbal y psicológica entre aquellas personas que comparten una jornada escolar. El conflicto en el ser humano ha estado presente desde su existencia; sin embargo, la solución a estos conflictos ha sido meritoria de ser estudiada y por ende mejoradas las vías para resolverlos, no obstante, la sociedad actual vive una crisis de tolerancia a raíz de la falta de valores en las familias, que perpetúa dichas problemáticas en el campo educativo, porque a la escuela se lleva lo que se aprende en casa. Por ello, se emprende este camino investigativo hacia la solución de la siguiente pregunta: ¿Cuál es la relación entre inteligencia emocional y los roles del maestro en la enseñanza y del estudiante en el aprendizaje, para el manejo de situaciones de conflicto en los grados $6^{\circ}$ en Instituciones Educativas Rurales del municipio de Rionegro? 
Es necesario que la escuela reconozca su papel en el desarrollo de habilidades emocionales para emprender la tarea de implementar acciones que lleven al afianzamiento de la inteligencia emocional en los escolares, de modo que la labor de orientar el aprendizaje se facilite con el mejoramiento de dichas habilidades, además el docente como dinamizador de los aprendizajes, está en el deber de conocer y manejar sus emociones y las de sus estudiantes para generar espacios pedagógicos que conlleven, a través de la experiencia, al mejoramiento y control de reacciones frente a la solución de conflictos, por ello surge el siguiente problema científico: ¿Cuál es la relación entre inteligencia emocional y los roles del maestro en la enseñanza y del estudiante en el aprendizaje, para el manejo de situaciones de conflicto en los grados $6^{\circ}$ en Instituciones Educativas Rurales del municipio de Rionegro? Así, la intención fue lograr Establecer la relación entre inteligencia emocional y los roles del maestro en la enseñanza y del estudiante en el aprendizaje, para el manejo de situaciones de conflicto en los grados $6^{\circ}$ en Instituciones Educativas Rurales del municipio de Rionegro. Para lo cual fue necesario trazar y cumplir cuatro objetivos específicos:

- Analizar el rol del maestro en la enseñanza en cuanto a la inteligencia emocional para la promoción de la tolerancia y el manejo del conflicto en el aula.

- Analizar el rol del estudiante en el desarrollo de la inteligencia emocional para el logro de la tolerancia y el manejo del conflicto en el aula.

- Medir los niveles de inteligencia emocional en estudiantes de Instituciones Educativas Rurales de Rionegro y su alcance en la solución de conflictos en el aula.

- Sugerir acciones pedagógicas pertinentes para la promoción de la tolerancia y el manejo del conflicto en el aula.

Teniendo como hipótesis: El rol que asumen los maestros en la enseñanza y el estudiante en el aprendizaje en situaciones de conflicto, influye en el desarrollo de la inteligencia emocional de los estudiantes.

En el rastreo bibliográfico, se esbozan y entretejen los conceptos que atañen a la presente investigación, donde se define la escuela desde diferentes autores acerca de la realidad educativa y a las vivencias diarias que tienen que ver con las relaciones y las expresiones de inteligencia emocional.

Desde la década de los 90's cuando Peter Salovey, profesor y rector de la Universidad de Yale y Jhon Mayer profesor de la Universidad de New Hamsphire, crean el término que luego daría la vuelta al mundo gracias a la popularidad del libro de Daniel Goleman. Dentro del campo de la IE, el grupo de investigación de Salovey y Mayer ha realizado, el mayor esfuerzo en desarrollar una visión científica del concepto sobre Inteligencia Emocional (IE) y ha tratado de ir resolviendo sistemáticamente las inevitables incógnitas que se plantean cuando surge un nuevo enfoque de investigación. Estos dos autores definen la Inteligencia Emocional como la habilidad de las personas para percibir (en uno mismo y en los demás) y expresar las emociones de forma apropiada, la capacidad de usar dicha información emocional para facilitar el pensamiento, de comprender y razonar sobre las emociones y de regular las emociones en uno mismo y en los demás. 
Estos autores acuñaron el término en 1990 (Salovery, Brackett \& Mayer, 2004) y han desarrollado un modelo teórico de IE bien contrastado en años posteriores. Además, fue este grupo quien se interesó muy pronto por los métodos de evaluación del concepto de IE proponiendo la primera herramienta de evaluación de auto-informe de IE. Desarrollaron formas de medición más objetivas que correspondería a una inteligencia genuina, su principal objetivo fue medir la IE como una inteligencia clásica tal como la inteligencia lógico-matemática o verbal, es decir, mediante tareas de ejecución que el sujeto debe realizar, supliendo así los problemas de sesgos que presentan los cuestionarios. El planteamiento de las medidas de habilidad es que para evaluar si una persona es hábil o no en un ámbito, en este caso el emocional y afectivo, la mejor forma de hacerlo es comprobar sus habilidades a través de diferentes ejercicios que requieran poner a prueba tales habilidades comparando posteriormente sus respuestas con criterios de puntuación predeterminados y objetivos, $\mathrm{y}$ son precisamente los conceptos de estos autores los que servirán como marco de referencia para la presente investigación, dado que en las últimas décadas ha impactado significativamente el campo laboral y educativo del ser humano.

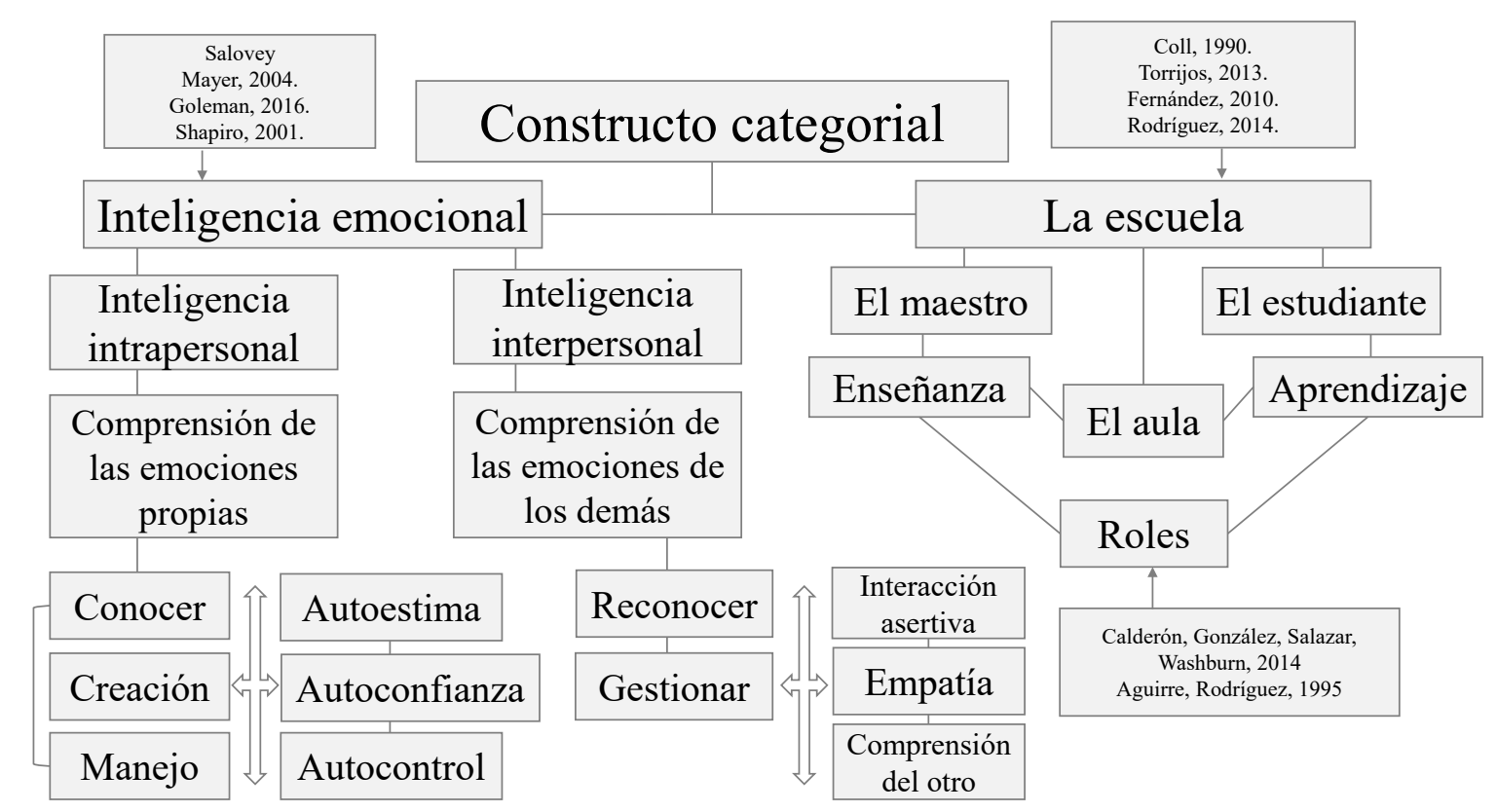

Figura 1. Constructo categorial

Fuente: elaboración propia

\section{METODOLOGÍA}

Este proyecto de investigación es de orden mixto, se fundamenta en el método inductivo. Tal como lo plantean los autores, los métodos, representan un conjunto de procesos sistemáticos empíricos y críticos de investigación (Hernandez Sampieri, Fernandez Collado \& Baptista Lucio, 2010) el cual articula los enfoques cualitativo y cuantitativo para desarrollar el estudios de los significados de las acciones humanas y de la vida social, para ello se pretende establecer la relación entre inteligencia emocional y los roles del maestro en la enseñanza y del estudiante en el aprendizaje para el manejo de situaciones de conflicto en los grados $6^{\circ}$ en Instituciones Educativas Rurales del municipio de Rionegro. De esta 
manera, este trabajo lleva consigo, la implementación, recolección y análisis de datos de orden cualitativo y cuantitativo para su discusión conjunta, logrando así un enriquecimiento de la información que permite comprender ampliamente el contexto estudiado.

Esta investigación se caracteriza por la implementación de un diseño flexible, para enfrentar la realidad en las poblaciones objeto de estudio. El nivel de profundización es de orden analítico, orientado a estructuras teóricas porque pretende interpretar sensiblemente datos complejos, pero con la rigurosidad que implica la ciencia; esto sin duda alguna orientado por un tipo de investigación mixta ya que se desarrollará para entender el fenómeno social del manejo de las emociones en los estudiantes y el papel que tiene la escuela al respecto, a través de la recolección de datos cualitativos y cuantitativos que permiten ampliar la perspectiva al investigador, así como la claridad, la riqueza y la variabilidad en la información, que rompe con la investigación uniforme. Así, la investigación se hace mixta en la medida en que se utilizan datos cuantitativos para llegar al análisis final; consecuente con el planteamiento se aplicación de instrumentos de carácter cuantitativo y cualitativo, en el primero la encuesta para la recolección de datos necesarios en la comprensión de la inteligencia emocional en los estudiantes del grado sexto, en el segundo la entrevista con los docentes para comprender el rol en situaciones de conflicto y en el desarrollo emocional de sus estudiantes. Esta relación entre paradigmas, permite ahondar con mayor subjetividad en la población seleccionada, la cual se elige usando las reglas aportadas por la estadística científica. La recolección de datos se realiza mediante la implementación de tres instrumentos que llevaron al cumplimiento de cada uno de los objetivos específicos, los cuales fueron la entrevista, el talle investigativo y el cuestionario que mide la inteligencia emocional.

\section{RESULTADOS Y DISCUSIÓN}

Los resultados y los hallazgos de esta investigación hacen parte de la realidad social que vive el municipio de Rionegro Antioquia actualmente, la constante en la mayoría de los directivos cuando responden el instrumento diagnóstico, sobre el comportamiento de los jóvenes del grado sexto, es corroborada en los demás instrumentos aplicados, una problemática seria de extra edad y comportamientos donde se comprueba la falta de inteligencia emocional en los estudiantes de los grados participantes en la investigación. Ello se vió reflejado a través de la presencia de desmotivación por el aprendizaje y conflictos entre compañeros en todas las instituciones investigadas y maltrato verbal, físico, hurto, falta de empatía y agresiones con los docentes, presentadas en un porcentaje significativo de las demás instituciones; lo que muestra una falta de vivencia de valores que lleva a los jóvenes a comportarse de manera inadecuada en los espacios de aprendizaje durante la jornada escolar; se observa un porcentaje mínimo de docentes que pierden el autocontrol a la hora de resolver los conflictos con sus estudiantes, donde se evidenciaron agresiones verbales entre docentes y estudiantes, haciendo necesaria la formación de la Inteligencia emocional para el mejoramiento de estrategias aplicadas en la resolución de conflictos en las instituciones rurales de Rionegro, ello lleva a denotar que otras investigaciones afines podrían aportar significativamente a la educación rural de Rionegro, asunto que sin duda, puede ser abordado igualmente en la población urbana, e incluso en otras localidades de la nación, ya que es un tema que hace parte de la formación de un ser humano integro, con habilidades que son necesarias en los diferentes ámbitos y campos donde se desarrolla la persona (familia, 
colegio, empresa, espacios sociales) y donde se debe enfrentar constantemente a la resolución de conflictos propios y en diferentes casos, conflictos de aquellas personas que están a su alrededor.

El taller investigativo, permite el acercamiento al estudiante y a la forma como asume su rol en el momento de resolver un conflicto, el instrumento central: "cuestionario para medir la inteligencia emocional" evaluó a cada uno de los jóvenes intervenidos, arrojando porcentajes concretos y medibles frente a las habilidades de autoconciencia, autocontrol, empatía, motivación y capacidad de trabajo en equipo, que son aquellas que fortalece la persona y que miden su nivel de inteligencia emocional. Para el caso de Rionegro en la habilidad de autoconciencia, se obtiene un promedio en las instituciones visitadas de 24,1 puntaje que deja algo de conformismo si se piensa en que el nivel es medio, pero también una intranquilidad si se tiene en cuenta que este nivel medio tiene su corte en 36 lo que representa un $66,9 \%$ del total, donde el puntaje promedio más bajo lo obtuvo la Institución Educativa Barro Blanco con 23,2 y la más alta fue Las Cuchillas con 25,1.

El taller posibilita la evaluación principalmente de la capacidad de evaluarse a sí mismo y ser crítico respecto a las acciones propias, relacionadas a los aciertos, desaciertos, sentimientos y emociones que surjan respecto a estos y cómo controlarlos o encauzarlos, situaciones que surgen al perder, o al hacer las cosas bien, ante la crítica de los demás; y según el resultado, se puede deducir que esta habilidad debe ser seriamente afianzada en los estudiantes ya que reflejan puntajes en el punto medio, pero más cercanos al bajo que al nivel alto. Conocer dicho análisis permite a cada institución centrar la atención según su puntaje, para implementar acciones que lleven al mejoramiento de la habilidad de la autoconciencia.

Frente a la habilidad del autocontrol se refleja el puntaje más bajo de los 5 tópicos con un promedio de 22,4 como resultado que representa un porcentaje de $62,3 \%$, con la I.E Domingo Savio con un resultado de 23,5 como el más alto y el más bajo es de 22,1 que se presenta en Santa Bárbara y en Guillermo Gaviria. Esta habilidad está centrada en la capacidad de tomar decisiones y controlar emociones, la autonomía, las formas de disipar los problemas a través del diálogo y el consenso, de buscar apoyo en los demás ante situaciones adversas o la aceptación de los demás en determinadas circunstancias. Se considera como un promedio bajo y se determina como un fenómeno que altera la inteligencia emocional de los estudiantes; preocupante el tema del autocontrol, porque es la habilidad que las personas deben manejar inicialmente como individuos, para adquirir otras habilidades que le permiten el trabajo con otras personas; es decir, con los estudiantes de los grados sextos de Rionegro, deben emprender acciones que los lleve a mejorar su capacidad de toma de decisiones, su autonomía, sus reacciones frente a los conflictos para que su nivel de inteligencia emocional mejore.

La habilidad de la motivación o aprovechamiento emocional cuenta con el promedio más alto de los tópicos con 26,1 de las respuestas, lo que representa un 72,5\%, donde Santa Bárbara presenta 27,4 como resultado más alto y Guillermo Gaviria obtuvo 25,3 de resultado que es el más bajo. Este parámetro está ligado a la motivación, la autoestima, el acompañamiento familiar o externo y el beneficio que esto pueda conllevar, la actitud con que se afrontan las normas y si carece de trascendencia acciones como hurtos o falta de respeto y maltrato a los demás, los deberes, las responsabilidades, si esta actitud es permeable y fácilmente por circunstancias irrelevantes se disipa o si por el contrario a pesar de 
factores externos esta actitud permanece férrea. Los resultados contrastan con la situación de conflicto percibida anteriormente de falta de motivación, por lo que se determina que los factores que originan los conflictos y las situaciones no deseadas están en otros ámbitos y la falta de motivación es una consecuencia más que un origen.

La empatía arroja un promedio de 24 con un porcentaje de $66,6 \%$, con Barro Blanco como el más bajo con el puntaje de 22,7 y Santa Bárbara el más alto con 25,1. La empatía evalúa la capacidad de estrechar lazos con los semejantes a través de la interpretación de las necesidades de estas personas y la adecuada actuación, de escuchar, de tolerar, además de la concepción que se tiene de los demás, si se considera que solo tienden a hacer daño y resultan indeseables o si son necesarios porque aportan positivamente a la vida. Cabe resaltar que las dos instituciones con menor empatía son las que fueron percibidas previamente con un conflicto relacionado con ignorar a los demás. Asimismo, las tres instituciones con menor promedio se había vislumbrado un conflicto en la falta de empatía entre compañeros. De esta manera, cada institución va generando un perfil que la representa frente a la investigación con aspectos específicos por atender, sin embargo, se considera que, según los puntajes, todas las instituciones tienen necesidades frente al afianzamiento de la inteligencia emocional.

Las habilidades sociales o capacidad de trabajo en equipo, tiene un promedio de 24,4 que representa un porcentaje de 67,7\%, con Santa Bárbara el puntaje más alto con 25,2 como promedio y Domingo Savio el más bajo con 23,5. Lo que devela el estado de las capacidades para pertenecer a grupos, de demostrar los sentimientos propios, de compartir y permitir que los demás sean parte fundamental de la vida diaria, de trabajar en equipo, de comprender a los demás y de querer pertenecer a un conjunto donde se comparta, se aprenda mutuamente y se establezcan relaciones entre diversas individualidades.

El promedio general de las instituciones rurales de Rionegro es de 121, el cual implica que, aunque el puntaje denote un punto medio con resultados positivos, se encuentra muy cerca de un punto bajo, por lo cual es necesaria la reflexión respecto al estado de la inteligencia emocional de los estudiantes ya que este factor es determinante para el rendimiento académico. Además, implica una incapacidad para evaluarse a sí mismo de manera adecuada y descubrir las buenas o malas actuaciones y cómo redirigir la situación o permanecer en esa dirección, determina un manejo de las emociones pobre, con baja asimilación del fracaso y las adversidades, poca motivación y falta de acompañamiento familiar, así como una incapacidad generalizada por seguir las normas y así poder construir entornos armoniosos donde se comprenda y tolere a los demás, sin la presencia de hurto o maltrato físico, desinterés ante las emociones y situaciones de las demás personas, por ende incapacidad para actuar en procura de ayudarlos, deficiencias en las relaciones sociales y trabajo en equipo que derivan en retraimiento y conductas solitarias, que muchas veces impiden la resolución de problemas académicos o personales, ya que no se recurre a la ayuda que los demás puedan brindar, lo que además genera conflictos al no permitir que lo externo se inmiscuya en la propia vida y sucesos tales como críticas o muestras de interés terminen como algo invasivo.

Las habilidades evaluadas, son un tema de vivencia cotidiana, no podría decirse que en alguna área se van a incluir temáticas que desarrollen alguna habilidad, porque se trata de la aplicación de estas en cada acción emprendida, desde el hogar, la escuela y los ambientes 
sociales, esto complejiza la tarea de la escuela porque se trata de la formación de un ser humano integro lo cual no puede ser representado a través de contenidos, sino que se materializa en las diferentes formas como se asumen o se resuelven los conflictos.

Durante la aplicación de la entrevista a los docentes, surgen datos relevantes que confirman la información de la encuesta inicial. Por ejemplo cuando se indagó por las reacciones de los estudiantes durante la resolución de un conflicto, los maestros manifiestan que inmediatamente hay presencia de desmotivación y abandono de la actividad académica que se esté realizando ya que hay que ofrecer al estudiante el espacio para bajar la tensión, la ira o la tristeza manifestadas a través del llanto o la melancolía o la agresión física y verbal, muchos de los estudiantes reaccionan con actos agresivos contra los demás, e incluso, contra ellos mismos, lo que deja ver la falta de tolerancia y la impulsividad a la hora de solucionar los conflictos que se presentan en el aula. Para ello, el docente recurre inicialmente al retorno de la calma del individuo o del grupo si es necesario, luego al diálogo como estrategia de expresión y escucha de sentimientos y si es necesario busca ayuda con sus directivos o con el maestro orientador. Procesos con dificultades mayores han sido remitidos a los comités de convivencias existentes en las instituciones, quienes se encargan de velar por un ambiente sano en los espacios escolares. Estas manifestaciones claramente expresadas por los docentes, dejan ver la necesidad del manejo adecuado en la resolución de conflictos en el aula, si bien, los maestros buscan estrategias para abordar las problemáticas presentes, sería muy enriquecedor capacitar a la comunidad en general sobre dicha temática con el fin de que sea el mismo estudiante, quien a través del autocontrol y la autoconciencia aborde la solución al conflicto sin llegar al estado de desmotivación y abandono de la actividad académica, que se convierte en una de las principales en el desarrollo del currículum, estos resultados corroboran los trabajos de Salovery, Brackett \& Mayer (2004) cuando concluyen que los profesores se encuentran por lo general motivados para realizar este tipo de formación. Además, para el docente de Rionegro, también se hace necesaria una formación en Inteligencia Emocional, que le ayuden a mejorar sus habilidades de autocontrol, autoconciencia, empatía, motivación y trabajo en equipo con el fin de que su labor se fundamente en las sanas relaciones con sus colegas, con los estudiantes y con la comunidad en general.

Los resultados de la presente investigación coinciden con los de Pacheco \& Berrocal (2004) cuando afirman que la evaluación de la IE en el aula supone una valiosa información para el docente en lo que respecta al conocimiento del desarrollo afectivo de los alumnos y permite un acercamiento efectivo al proceso académico, de esta manera se concibe necesaria la evaluación de la IE en el aula como herramienta para el docente en el momento de realizar su planeación. Para (Palomera, Fernandez-Berrocal \& Bracckett, 2008) se hace indispensable formar al docente en el tema de IE con el fin de evitar secuelas emocionales y de salud y principalmente para que su orientación con los estudiantes este encaminada hacia el adecuado manejo de las emociones; para ellos, esta formación se debe dar desde las universidades y la formación del docente como profesional, asunto que se reflejó en los resultados de esta investigación, porque los mismo docentes manifestaron en sus entrevistas la importancia de ser ellos, el ejemplo a seguir, por lo tanto, deben estar fortalecidos en el tema de la IE, claro está, que se plantea como una posibilidad desde las capacitaciones que se ofrecen a los docentes a nivel administrativo. 
En el contexto escolar, los educadores son los principales referentes emocionales de sus alumnos. La capacidad del profesor para captar, comprender y regular las emociones de sus alumnos es el mejor índice del equilibrio emocional de su clase, así lo plantean (Fernández-Berrocal \& Extremera, La inteligencia emocional como una habiidad esencial en la escuela., 2011) idea que se comparte desde los resultados de la presente investigación, cuando se encuentra que es el docente el modelo o referente a seguir por parte de los estudiantes, lo que implica un equilibrio emocional en la clase si se cuenta con un docente asertivo o un caos emocional si el docente carece de habilidades emocionales.

Por su parte (Fernández-Berrocal \& Extremera, 2011) asumen las habilidades integradas en su modelo como, habilidades de obligatoria enseñanza en la escuela, asunto que dista un poco de esta propuesta, donde se concibe la orientación de las habilidades para el manejo de las emociones como un asunto transdisciplinar que debe ser tenido en cuenta por todos los docentes de todas las áreas en el diseño de sus estrategias para la orientación de las diferentes espacios de aprendizaje; es decir, la adquisición de habilidades para el manejo de la inteligencia emocional debe darte desde las vivencias diarias, desde las experiencias cotidianas, desde la práctica, contando con el apoyo de todos los docentes, asunto que es apoyado por (Ibarrola, 2003) Desde su investigación: "Dirigir y educar con inteligencia emocional".

Algo positivo que se encontró fue cuando se indagó por los tipos de relaciones que se tejen entre los estudiantes, los docentes manifestaron las diferentes formas como se relacionan los jóvenes a través de la amistad, los noviazgos, las relaciones cordiales entre los docentes y con los estudiantes, en algunos casos la presencia de enemistades, sin embargo aclaraban que se trata de asuntos transitorios y cotidianos que son resueltos con brevedad; esto indica que los conflictos que se presentan en los estudiantes de las instituciones rurales de Rionegro Antioquia son resueltas en el momento que se presentan y no dejan huellas permanentes en la población, lo que debe llevar, con mayor razón al fortalecimiento de estrategias con la comunidad educativa que ayuden a estos jóvenes a mejorar el manejo que le dan a los problemas que se les presentan, y ello podría lograrse a través del trabajo con padres de familia específicamente, quienes son aquellos formadores iniciales de las habilidades que ayudan en la resolución de conflictos, es claro que no tienen una formación específica para ello, sin embargo, cada acción emprendida en la familia, será la que el estudiante refleje en los demás espacios, así que, seria enriquecedor llevar estos padres de familia a talleres donde se les trabaje no solo la teoría de la inteligencia emocional, sino también actividades prácticas a partir de sus vivencias cotidianas y la forma como se deber resolver, siendo muy coherentes en que prima la integridad y el bienestar de quienes se confrontan en un conflicto.

Para los maestros es claro que existen estrategias efectivas durante la resolución de conflictos y no tienen duda en manifestarlas a la hora de abordarlos con la pregunta de la entrevista: ¿Qué debe hacer un maestro para lograr mayor nivel de tolerancia en los estudiantes en las situaciones de conflictos en el aula? Para la cual surgen respuestas como: enseñar a los estudiantes la habilidad de la resiliencia, la paciencia tanto como habilidad para ellos como para los mismos docentes, a través de la cual enlazamos el diálogo como estrategia permanente, (siendo esta la más recurrente en las respuestas), y mediante la cual se permite escuchar y redireccionar la problemática a través de la solución más adecuada. Cumplir con el proceso que tiene estipulado cada manual de convivencia y ser un mediador, es de las respuestas recurrentes que manifiestan los maestros cuando se les aborda por el uso de estrategias en la resolución de conflictos, todas las respuestas llevan un trasfondo y es la 
formación de un ser humano integro, es la formación del ser antes que del hacer, sin olvidar que todas las competencias tienen una función fundamental en el individuo, los docentes expresan la necesidad de formar en valores, en el respeto y la tolerancia para lograr impactar la sociedad que se refleja hoy día en los adultos. Se encuentra una constante cuando se habla con el maestro y es el reconocimiento del ejemplo como fuerza que arrastra a los jóvenes, manifestando que los profesionales de la educación son seguidos por sus estudiantes, ya sea de manera positiva o de manera negativa; otras estrategias importantes aparecen en las respuestas de los maestros, como lo son, la comunicación asertiva, el abordaje de los conflictos a través del plan de aula, la implementación de charlas motivacionales y de valores, el trabajo constante de la tolerancia y el respeto por el otro.

Cuando se inicia el proceso de investigación, se piensa en un tema de tesis y de necesidad en el campo que se decide investigar, seguidamente se plantean unos objetivos que direccionan el camino a seguir; los planteados en la presente investigación son resueltos con éxito, porque se logra indagar, inicialmente, la necesidad que tienen las instituciones rurales por ser objeto de investigación en cuanto a la problemática latente en los grados sextos, a través de sus directivos y por medio de una sencilla encuesta, lo que permitió dar el paso siguiente, ya que se comprueba parte de los supuestos iniciales, donde se considera la necesidad de las instituciones rurales de ser estudiadas. Esta rápida relación abre las puertas para la aplicación del taller investigativo que es el instrumento que lleva a la resolución de dos objetivos específicos: Analizar el rol del estudiante en el desarrollo de la inteligencia emocional para el logro de la tolerancia y el manejo del conflicto en el aula; y medir los niveles de inteligencia emocional en estudiantes de Instituciones Educativas Rurales de Rionegro y su alcance en la solución de conflictos en el aula, a través del desarrollo de varias actividades, pero sobre todo mediante la aplicación del test: "Cuestionario para evaluar la Inteligencia Emocional" construido y validado por el doctor Chiriboga, y el análisis de casos similares a los que viven en su cotidianidad.

Otro de los objetivos cumplidos es analizar el rol de docente en el desarrollo de la Inteligencia Emocional, lo que se logra a través de una entrevista, donde el maestro aporta significativamente con el proceso de investigación, si bien, fue el punto coyuntural, debido a las múltiples ocupaciones de los docentes, se logra el objetivo de revisar el rol y la forma como se abordan los conflictos en el aula y con el valor agregado, de los aportes en estrategias aplicables en los estudiantes para el manejo adecuado en la resolución de conflictos, lo que se convierte en un aporte para el logro del último objetivo específico: sugerir acciones pedagógicas pertinentes para la promoción de la tolerancia y el manejo del conflicto en el aula.

Reunir toda esta información, procesarla e interpretarla, permite el alcance y el proceso hermenéutico que llevan a la resolución del objetivo general, planteado inicialmente con tanta ambición, y modificado en el camino, según las necesidades que surgieron: Establecer la relación entre inteligencia emocional y los roles del maestro en la enseñanza y del estudiante en el aprendizaje, para el manejo de situaciones de conflicto en los grados $6^{\circ}$ en Instituciones Educativas Rurales del municipio de Rionegro. Ahora, la responsabilidad social como investigadora, es llevar los hallazgos a las comunidades visitadas, e incluso a las que no quedaron en la muestra, para aportar, de alguna manera, en el proceso de formación del individuo rionegrero. 
El proceso que se vivió durante la investigación, permite corroborar la hipótesis planteada inicialmente: El rol que asumen los maestros en la enseñanza y el estudiante en el aprendizaje en situaciones de conflicto, influye en el desarrollo de la inteligencia emocional de los estudiantes, porque como se menciona durante el análisis de la información, el ejemplo hace seguidores, y en el papel del maestro es común que los estudiantes actúen como ven a los adultos actuar, por ello a la hora de resolver un conflicto, el docente no solo debe ser mediador, sino también vivenciador de experiencias que muestren la formas sensatas de dialogar, comunicarse con asertividad, manejar el autocontrol y la autoconciencia, estar motivado frente a lo que hace y tener la capacidad de trabajar con sus colegas de manera cordial.

\section{CONCLUSIONES}

El conocimiento de sí mismo y las relaciones en sociedad, son habilidades necesarias que hacen parte de la formación integral del ser humano, y que se fortalecen durante el proceso educativo del individuo, perdurando durante todos los momentos en que éste se relaciona e interacciona en diferentes espacios sociales; esto indica que inevitablemente, la escuela está involucrada en desarrollo, afianzamiento y potencialización de habilidades que fortalecen el carácter y la Inteligencia Emocional, porque se convierte en la institución que aporta a la formación del ser, como agente activo de la sociedad, durante una cantidad de años significativa.

El rol que desempeña el maestro en el manejo adecuado de conflictos, es determinante; según la información analizada, los estudiantes ven en el maestro un ejemplo y continuamente actúan siguiendo las acciones que observan en él; ver el docente como referente, les hace seguir sus pasos (ya sean negativos o positivos) considerando que es la manera correcta de actuar. Este hallazgo compromete al maestro al adecuado manejo de las situaciones que emergen en el aula y en los espacios del conocimiento, usando como herramientas: el diálogo, la escucha y la comprensión de los estudiantes, siguiendo correctamente los procesos y las remisiones pertinentes según cada caso, para hacer posible los espacios de solución consensuada de las situaciones de conflicto que se puedan presentar y siendo ejemplo en sus relaciones intra e interpersonales, tal como quedó registrado en la evidencias recolectadas, tanto de estudiantes como de docentes.

El rol del estudiante se orienta a ser un sujeto que se encuentra en proceso de formación; lo que se evidencia como resultado de la información recolectada, es que sus niveles de Inteligencia Emocional no superan un nivel medio, y dentro de este nivel obtienen un puntaje promedio de 123, estando muy cercanos al nivel bajo, lo que indica que en los estudiantes del grado sexto de Instituciones Educativas Rurales de Rionegro se debe fortalecer significativamente los niveles de I.E. Aunque durante los talleres aplicados, manifestaron de manera escrita, soluciones factibles a los conflictos, como lo son: el diálogo, el acercamiento, la confianza con los adultos, la calma y el respeto; en las acciones que emprenden día a día, se les dificulta la relación con el otro y el conocimiento de sus emociones y las formas de reaccionar para tener control de éstas, esto quedó en evidencia durante el taller ya través de las entrevistas realizadas a los docentes, donde se manifestó que a la hora de resolver las dificultades que se les presentan con pares o superiores, se dejan llevar por las emociones negativas y reaccionan con violencia, con odios, rencores, tristeza, agresividad. 
El trabajo en la escuela de la autoconciencia, autorregulación, empatía, motivación y la capacidad de trabajo en equipo, se torna complejo, sin embargo, con la presente investigación se evidencia lo permeados que se encuentran todos los espacios del conocimiento con dicha formación, porque siempre se está en contacto con otros, lo que implica tener habilidades que ayuden a mantener relaciones exitosas para lograr a la vez el éxito personal. De esta manera se afirma que la escuela debe pensar e incluir entre sus planes de trabajo, estrategias que fortalezcan las habilidades propias de la Inteligencia Emocional, sin enfocarla o darle la responsabilidad a un área o asignatura en especial, sino como un trabajo transdisciplinar que permea todas las áreas y los espacios de interacción, con el fin de lograr que los conflictos que se presentan constantemente entre estudiantes, y en ocasiones con maestros y comunidad en general, sean resueltos a través del diálogo y la comunicación asertiva.

Se reconoce que la formación docente para la enseñanza en la educación básica, está enfocada en el desarrollo de habilidades académicas, intelectuales, éticas y metodológicas; por eso se hace necesaria, a la vez, la inclusión de contenidos que favorezcan la adquisición de conocimientos en cuanto a la educación emocional. Así mismo, como lo mencionan (Extremera \& Fernández-Berrocal, 2004), el éxito del profesorado queda también vinculado a desarrollar personas integradas a la sociedad, con herramientas sociales y emocionales que les permitan afrontar los desafíos de la vida cotidiana. Esto se puede relacionar a lo mencionado por los docentes participantes, los cuales expresan que, ante situaciones que se les presentan en el aula, en se quedan cortos en cuanto a qué hacer o cómo enfrentarlas. Es así como surgen reacciones de frustración, enojo y hasta decepción.

La aplicación de la escala de inteligencia emocional requiere de otras técnicas para encontrar explicaciones a los resultados, en este caso la aplicación de la entrevista y el taller brindaron dichos elementos que muestran condiciones reales que experimentan los sujetos participantes en contexto situado de la escuela y poder sugerir proyecciones en lo educativo e investigativo.

\section{REFERENCIAS}

Correa, J. I. (2018) Acercamiento conceptual a violencias, conflictos y cultura de paz. Relaciones en el contexto de la primera infancia. En Subjetividades y prácticas socioeducativas de los conflictos y las violencias en la educación infantil. Divergráficas. S.A.S. https://issuu.com/boletin_marcat_tdea/docs/libro_educacion_ infantil

Extremera, N., \& Fernández-Berrocal, P. (2004). La importancia de desarrollar la inteligencia emocional en el profesorado. Revista iberoamericana de educación, 33(8), 1-9. http://www.academia.edu/download/51176555/La_importancia_de_desarrollar_la_ intelig20170104-22267-10nk31.pdf

Fernández-Berrocal, P., \& Pacheco, N. E. (2002). La inteligencia emocional como una habilidad esencial en la escuela. Revista Iberoamericana de educación, 29(1), 1-6. https://rieoei.org/RIE/article/download/2869/3813

Goleman, D. (1999). La inteligencia emocional. Bantam Books. 
Hernandez Sampieri, R., Fernández Collado, C., \& Baptista Lucio, P. (2010). Metodología de la investigación. MG GrawHi Educación.

Ibarrola, B. (2003). Dirigir y educar con inteligencia emocional [ponencia]. VII Congreso de Educación y Gestión, Madrid.

Pacheco, N. E., \& Berrocal, P. F. (2004). La inteligencia emocional: Métodos de evaluación en el aula. Revista Iberoamericana de educación, 34(1), 1-12. https://rieoei.org/RIE/ article/download/2887/3820

Palomera, R., Fernández-Berrocal, P., \& Brackett, M. A. (2008). La inteligencia emocional como una competencia básica en la formación inicial de los docentes: algunas evidencias. Electronic journal of research in educational psychology, 6(2), 437-454. https://www.redalyc.org/pdf/2931/293121924010.pdf

Palomero, P.(2009). Desarrollo de la competencia social y emocional del profesorado: una aproximación desde la psicología humanista. Revista electrónica interuniversitaria de formación del profesorado, 12(2), 145-153. https://dialnet.unirioja.es/descarga/ articulo/3039102.pdf

Salovery, P., Brackett, M., \& Mayer, J. (2004). Emotional intelligence: Key readings on the Mayer and Salovery model. Publisher's Cataloging in publication.

Shapiro, L. E. (2001). La inteligencia emocional en los niños una guía para padres y maestros. Suma de letras S.L. 